\title{
Czech National Phytosociological Database
}

\author{
Milan Chytrý \& Dana Michalcová
}

\begin{abstract}
The Czech National Phytosociological Database (GIVD ID EU-CZ-001) was established in 1996 at Masaryk University in Brno. Currently it is hosted by the Department of Botany and Zoology of this university. It is an electronic archive of phytosociological relevés and related records of species composition from vegetation plots which have been sampled on the territory of the Czech Republic. The data stored in the database are used for the development of new national vegetation classification of the Czech Republic, biodiversity analyses at local, regional, national and international level, as a source of information about distribution of plant communities and species, and for developing and testing new methods in the analysis of large sets of vegetation-plot data.
\end{abstract}

Keywords: Czech Republic; phytosociology; plant community; relevé; vegetation plot.

GIVD Database ID: EU-CZ-001

Last update: $2012-05-08$

\section{Czech National Phytosociological Database}

Scope: All phytosociological relevés and related vegetation plot records from the Czech Republic.

Status: ongoing capture

Period: 1922-2011

Database manager(s): Milan Chytrý (chytry@sci.muni.cz); Dana Michalcová (danmich@sci.muni.cz)

Owner: Department of Botany and Zoology, Masaryk University, Brno, Czech Republic

Web address: http://www.sci.muni.cz/botany/vegsci/dbase.php?lang=cz

Availability: free upon request

Online upload: no

Online search: no

Database format(s): TURBOVEG

Export format(s): TURBOVEG

Publication: Chytrý M. \& Rafajová M. (2003): Czech National Phytosociological Database: basic statistics of the available vegetation-plot data. Preslia 75:1-15.

Plot type(s): normal plots Plot-size range: $0.09-10,000 \mathrm{~m}^{2}$

Non-overlapping plots: 99,586

Estimate of existing plots: 105,000

Completeness: $95 \%$

Total plot observations: 99,586

Number of sources: 2196

Valid taxa: 4,394

Countries: CZ: 100.0\%

Forest: [NA] - Non-forest: [NA]

Guilds: all vascular plants: $100 \%$; bryophytes (terricolous or aquatic): $60 \%$; lichens (terricolous or aquatic): $50 \%$; algae (terricolous or aquatic): $0 \%$; non-terricolous taxa (epiphytic, saxicolous, lignicolous): $2 \%$

Environmental data: altitude: $60 \%$; slope aspect: 59\%; slope inclination: 59\%; soil depth: $1 \%$; surface cover other than plants (open soil, litter, bare rock etc.): $13 \%$; soil $\mathrm{pH}: 1 \%$

Performance measure(s): presence/absence only: 0\%; cover: $100 \%$

Geographic localisation: GPS coordinates (precision $25 \mathrm{~m}$ or less): $11 \%$; point coordinates less precise than GPS, up to $1 \mathrm{~km}$ : $84 \%$; small grid (not coarser than $10 \mathrm{~km}): 4 \%$; political units or only on a coarser scale $(>10 \mathrm{~km}): 2 \%$

Sampling periods: 1920-1929: 0.2\%; 1930-1939: 0.7\%; 1940-1949: 1.6\%; 1950-1959: 2.8\%; 1960-1969: 9.5\%; 1970-1979: 13.6\%; 1980-1989: 13.3\%; 1990-1999: 22.5\%; 2000-2009: $28.4 \%$

Information as of 2012-07-12; further details and future updates available from http://www.givd.info/ID/EU-CZ-001

Milan Chytrý* (chytry@sci.muni.cz), Dana Michalcová (danmich@sci.muni.cz)

Department of Botany and Zoology, Masaryk University, Kotlárská 2, CZ-61137 Brno, CZECH REPUBLIC

*Corresponding author 